\title{
ENTRY AND RETURN TIMES DISTRIBUTION
}

\author{
NICOLAI HAYDN
}

\section{INTRODUCTION}

The area of dynamical systems is characterised by the study of longterm behaviour as evidenced by the quest to find conditions under which a map has an invariant measures or stable and unstable manifolds. If there is an invariant probability measure, then sensitivity of orbits on initial data expresses itself in ergodicity and mixing properties. One way to measure this is to look at the decay of correlation functions where two functions, one of them pulled back, are jointly integrated. This typically requires sufficient regularity of the functions. In a purely measure theoretic setting we have Poincaré's recurrence theorem (see below) which states that every point returns to itself in finite time. This result has subsequently been quantified in many different ways by Kac and others as we will describe below. This is the point of view we want to adopt in this paper where we review some results on entry and also return times in dynamical systems. In the process we will focus on the statistics which is dominated by long term independence. To quantify very short term returns where deterministic dependency dominates and which display different statistics is also currently worked on by various people but not considered in this review. Also not discussed here are zero entropy systems that can have many different 'non-standard' limiting distributions. Except for the second and last sections the systems considered here have positive entropy.

In this paper we discuss six aspects of return and entry times statistics as follows. In the second section we look at general entry and return times results which apply to any system with an invariant ergodic probability measure. In the third section we shortly discuss different kinds of mixing which play important roles in distribution results most of it require symbolic coding of orbits via measurable partitions. In the fourth section we look at results on the first entry and return times. We then proceed to review past results on the entry and return times distributions which are dominated by the approach of Galves and Schmitt from 1997. In the fifth section we review results on higher order returns which we categorise by the method that was used to obtain the distribution results. In the sixth section we look at distributions near periodic orbits and in the seventh section we look at recurrence times which measure the time it takes for a point to return to its own neighbourhood.

Previous reviews on this topic were done by Abadi and Galves [6] and Coelho [23.

Date: August 8, 2018. 


\section{RETURN/ENTRY TIMES AND THE INDUCED MAP}

Let $T$ be a map on a space $\Omega$ and assume $\mu$ is a $T$-invariant probability measure on $\Omega$. For a measurable set $U \subset \Omega$ we define the return times function

$$
\tau_{U}(x)=\min \left\{j \geq 1: T^{j} x \in U\right\}
$$

for $x \in U$ and we put $\tau_{U}(x)=\infty$ if the forward orbit of $x$ never intersects $U$. We assume $\mu(U)>0$.

2.1. Poincaré recurrence theorem. The earliest and most famous result on the return time is due to Poincaré [67] in 1890.

Theorem 1. (Poincaré recurrence theorem) Let $T: \Omega \rightarrow \Omega$ and $\mu$ be a T-invariant probability measures. If $\mu(U)>0$, then $\tau_{U}(x)<\infty$ for almost every $x \in U$.

This theorem is easily proven as follows: Let $U_{n}=\bigcup_{j=n}^{\infty} T^{-j} U$ for the set of points $x \in \Omega$ that enter $U$ at least once after time $n$. Obviously $U_{0} \supset U_{1} \supset U_{2} \supset \cdots$, and also $U_{n}=T^{-1} U_{n+1}$ which implies by the invariance of the measure that $\mu\left(U_{n}\right)=$ $\mu\left(T^{-1} U_{n+1}\right)=\mu\left(U_{n+1}\right)$ and consequently $\mu\left(U_{0}\right)=\mu\left(U_{n}\right) \forall n$. Now $W=\bigcap_{n=1}^{\infty} U_{n}=$ $\{x \in \Omega$ enters $U$ infinitely often $\}$ and $V=W \cap U=\{x \in U$ enters $U$ infinitely often $\}$. Since $\mu\left(U_{0}\right)=\mu\left(U_{n}\right)$ we obtain that $\mu(W)=\mu\left(U_{0}\right)$ and since $U \subset U_{0}$ we conclude that $\mu(V)=\mu(U)$.

The recurrence statement does not extend to infinite measures as the example $T x=$ $x+1$ on the real line shows where no set of positive measure is recurrent.

2.2. Kac's theorem. For $U \subset \Omega, \mu(U)>0$ Poincaré's recurrence theorem states that $\tau_{U}(x)<\infty$ for almost every $x \in U$, however it doesn't tell us anything about how big $\tau_{U}$ is. Assuming ergodicity, a well known theorem by Kac from 1947 tells us what the average value is, i.e. the expected value of $\tau_{U}$ on $U$ and in particular also asserts that $\tau_{U}$ is integrable on $U$.

Theorem 2. 53] If $\mu$ is an ergodic $T$-invariant probability measure on $\Omega$ then for any $U \subset \Omega$ of positive measure one has

$$
\int_{U} \tau_{U}(x) d \mu(x)=1
$$

This theorem is a consequence of the Birkhoff ergodic theorem but can also be proven in other ways as well. However in the invertible case it is easy to see why the result is true and involves the construction of a Kakutani tower. Put $U_{k}=\left\{x \in U: \tau_{U}(x)=k\right\}$, $k=1,2, \ldots$, for the level sets of the return time. Then $U=\dot{\bigcup}_{k=1}^{\infty} U_{k}$ is a disjoint union and the sets $T^{j} U_{k}$ for $j=0,1, \ldots, k-1, k \in \mathbb{N}$, are pairwise disjoint. Since $\mu$ is ergodic, $\Omega=\bigcup_{k} \bigcup_{j=1}^{k-1} T^{j} U_{k}$ and as $T$ is invertible $\mu\left(T^{j} U_{k}\right)=\mu\left(U_{k}\right)$. We thus obtain the statement of Kac's theorem:

$$
1=\mu(\Omega)=\sum_{k=1}^{\infty} \sum_{j=0}^{k-1} \mu\left(T^{-1} U_{k}\right)=\sum_{k} k \mu\left(U_{k}\right)=\int_{U} \tau_{U} d \mu .
$$


If the function $\tau_{U}$ is extended to the entire space $\Omega$ then we refer to it at the entry times function. It is not necessarily integrable over all of $\Omega$. In fact $\tau_{U}$ is integrable over $\Omega$ if and only if it is $\mathscr{L}^{2}$-integrable over $U$.

2.3. Entry and return times distributions. Let $B \subset \Omega(\mu(B)>0)$ and consider the entry times function $\tau_{B}(x)$ where $x \in \Omega$. For (parameter values) $t>0$ put

$$
F_{B}(t)=\mathbb{P}\left(\tau_{B}>\frac{t}{\mu(B)}\right)=\mu\left(\left\{x \in \Omega: \tau_{B}(x)>\frac{t}{\mu(B)}\right\}\right)
$$

for the entry time distribution to $B$. Clearly, the entry times distribution $F_{B}(t)$ is locally constant on intervals of length $\mu(B)$ and has jump discontinuities at values $t$ which are integer multiples of $\mu(B)$. For any $s \in \mathbb{N}_{0}$ one has

$$
\left\{\tau_{B}>s+1\right\}=T^{-1}\left\{\tau_{B}>s\right\} \backslash T^{-1} B
$$

and consequently

$$
\mathbb{P}\left(\tau_{B}=s+1\right)=\mathbb{P}\left(\tau_{B}>s\right)-\mathbb{P}\left(\tau_{B}>s+1\right) \leq \mu(B)
$$

which shows that the jumps at the discontinuities are bounded by $\mu(B)$.

Correspondingly, for the return time function $\tau_{B} \mid B$, we call

$$
\tilde{F}_{B}(t)=\mathbb{P}_{B}\left(\tau_{B}>\frac{t}{\mu(B)}\right)
$$

the return times distribution.

Example. Let $\Omega$ be the shift space over an alphabet $\mathcal{A}$ (which is finite or countably infinite). That is $\Omega=\mathcal{A}^{\mathbb{Z}}$ and points $\vec{x}$ in $\Omega$ are of the form $\vec{x}=\left(\cdots x_{-2} x_{-1} \cdot x_{0} x_{2} \cdots\right)$, where $x_{j} \in \mathcal{A}$ and the dot indicates the 0 th coordinate. The map on $\Omega$ is the left shift transformation $\sigma$ given by $\left(\sigma(\vec{x})_{j}=x_{j+1} \forall j\right.$. If $B=U\left(x_{0} x_{1} \cdots x_{n-1}\right)=\{\vec{y} \in \Omega$ : $\left.y_{0} \cdots y_{n-1}=x_{0} \cdots x_{n-1}\right\}$ is an $n$-cylinder then entry time $\tau_{B}(\vec{y})$ for $\vec{y} \in \Omega$ measures the 'time' it take to see the word $x_{0} x_{1} \cdots x_{n-1}$, that is

$$
\tau_{B}(\vec{y})=\min \left\{j \geq 1: y_{j} \cdots y_{j+n-1}=x_{0} \cdots x_{n-1}\right\} .
$$

On the other hand if we restrict to $\vec{y} \in B$ then $\tau_{B}$ is the return time and measures the time it takes for the initial $n$-word to reappear. That is

$$
\tau_{B}(\vec{x})=\min \left\{j \geq 1: x_{j} \cdots x_{j+n-1}=x_{0} \cdots x_{n-1}\right\} .
$$

The function $\tilde{F}_{B}(t)$ then measures the probability to see the first $n$-word again after rescaled time $t / \mu(B)$.

The following result relates the limiting entry times distribution to the limiting return times distribution. It turns out that a simple formula allows us to compute one from the other one.

Lemma 3. For any $B \subset \Omega(\mu(B)>0)$ let $F_{B}^{\prime}$ be the right sided derivative of the largest continuous piecewise linear function that lies below $F_{B}$ and is linear on the intervals $[k \mu(B),(k+1) \mu(B)], k \in \mathbb{N}$. Then

$$
-F_{B}^{\prime}(t)=\tilde{F}(t)
$$


Proof. We have that $F_{B}(t)=\mathbb{P}\left(\tau_{B}>t / \mu(B)\right), \tilde{F}_{B}(t)=\mathbb{P}_{B}\left(\tau_{B}>t / \mu(B)\right)$ and for $j=0,1,2, \ldots$ put $A_{j}=\Omega \backslash T^{-j} B=T^{-j} B^{c}$ and $(j \leq k) D_{j}^{k}=\bigcap_{\ell=j}^{k} A_{\ell}=\left\{x \in \Omega: T^{\ell} x \notin\right.$ $B \forall \ell=j, \ldots, k\}$. Then for any $s \in \mathbb{N}$

$$
\left\{x \in \Omega: \tau_{B}(x)=s\right\}=T^{-s} \cap D_{1}^{s-1}=D_{1}^{s-1} \backslash D_{1}^{s}
$$

and hence

$$
\mathbb{P}\left(\tau_{B}=s\right)=\mu\left(D_{1}^{s-1}\right)-\mu\left(D_{1}^{s}\right)=\mu\left(D_{0}^{s-2}\right)-\mu\left(D_{0}^{s-1}\right)
$$

by invariance of $\mu$ as $D_{1}^{s}=T^{-1} D_{0}^{s-1}$. Since we also have

$$
\mu\left(D_{0}^{s-2}\right)=\mu\left(D_{1}^{s-1}\right)=\mu\left(B \cap D_{1}^{s-1}\right)+\mu\left(D_{0}^{s-1}\right),
$$

we consequently get

$$
\mu\left(\left\{x \in B: \tau_{B}(x)>s-1\right\}\right)=\mu\left(B \cap D_{1}^{s-1}\right)=\mathbb{P}\left(\tau_{B}=s\right) .
$$

On the other hand, if we put $s=\frac{t}{\mu(B)}$, then we can also write

$$
\begin{aligned}
\mathbb{P}\left(\tau_{B}=s\right) & =\mathbb{P}\left(\tau_{B}>s-1\right)-\mathbb{P}\left(\tau_{B}>s\right) \\
& =\mathbb{P}\left(\tau_{B}>\frac{t}{\mu(B)}-1\right)-\mathbb{P}\left(\tau_{B}>\frac{t}{\mu(B)}\right) \\
& =F_{B}(t-\mu(B))-F_{B}(t) \\
& =-\mu(B) F_{B}^{\prime}(t-\mu(B)) .
\end{aligned}
$$

Combining this with the previous identity for $\mathbb{P}\left(\tau_{B}=s\right)$ yields

$-F_{B}^{\prime}(t-\mu(B))=\frac{\mu\left(\left\{x \in B: \tau_{B}>(t-\mu(B)) / \mu(B)\right\}\right)}{\mu(B)}=\mathbb{P}_{B}\left(\tau_{B}>\frac{t-\mu(B)}{\mu(B)}\right)=\tilde{F}_{B}(t-\mu(B))$.

Now let $B_{n} \subset \Omega\left(\mu\left(B_{n}\right)>0\right)$ be a sequence of subsets so that $\mu\left(B_{n}\right) \rightarrow 0^{+}$as $n \rightarrow \infty$. If the limit $F(t)=\lim _{n \rightarrow \infty} F_{B_{n}}(t)$ exists for almost every $t \in(0, \infty)$ then we say $F$ is the limiting entry times distribution. Similarly $\tilde{F}=\lim _{n \rightarrow \infty} \tilde{F}_{B_{n}}(t)$ is the limiting return times distribution if the limit exists almost surely. Note that the limiting entry times distribution $F$ is Lipschitz continuous with Lipschitz constant 1 . The limiting return times distribution however does not have to have such regularity. If we apply the last lemma to the sets $B_{n}$ and take a limit then we obtain the following translation formula (from 2005).

Theorem 4. [47] Let $B_{n} \subset \Omega\left(\mu\left(B_{n}\right)>0\right)$ be a sequence of sets so that $\mu\left(B_{n}\right) \rightarrow 0^{+}$. If the limits $F(t)=\lim _{n \rightarrow \infty} F_{B_{n}}(t), \tilde{F}(t)=\lim _{n \rightarrow \infty} \tilde{F}_{B_{n}}(t)$ exist (pointwise) then

$$
F(t)=1-\int_{0}^{t} \tilde{F}(s) d s .
$$

Since the limiting return distribution $\tilde{F}(t)$ is monotonically decreasing to zero, one sees that $F(t)$ is convex. We cannot a priori assume that $\int_{0}^{\infty} \tilde{F} d s$ is equal to 1 . This is still an open question at this point.

We also observe that the limiting entry times distribution and return times distribution are the same only if they are exponential, that is $\tilde{F}=F$ if only if $F(t)=\tilde{F}(t)=e^{-t}$. This 
result was proven independently in [52] but follows very easily now from the translation formula. A somewhat generalised version of the theorem was proven in [8].

Lacroix has shown that if $F(t)$ is an eligible limiting distribution, that is it satisfies $F(0)=1$, is continuous, convex, monotonically decreasing on $(0, \infty)$ and $F(t) \rightarrow 0^{+}$as $t \rightarrow \infty$, then for any ergodic $T$-invariant probability measure $\mu$ there exists a sequence of positive measure sets $B_{n} \subset \Omega$ so that $\mu\left(B_{n}\right) \rightarrow 0$ and such that $F(t)=\lim _{n \rightarrow \infty} F_{B_{n}}(t)$ for every $t \in(0, \infty)$. Of course, the sets $B_{n}$ are typically pretty wild looking and in particular they are not topological balls or cylinder sets (if there is a partition).

2.4. Partitions. Let us assume that $\Omega$ has a measurable partition $\mathcal{A}$ which we assume to be generating. Then $\mathcal{A}^{n}=\bigvee_{j=0}^{n-1} T^{-j} \mathcal{A}$ is its $n$th join and for a point $x \in \Omega$ we put $A_{n}(x)$ for the unique $n$-cylinder in $\mathcal{A}^{n}$ which contains $x$. Let us put $F_{x}^{n}(t)=\mathbb{P}\left(\tau_{A_{n}(x)}>\right.$ $\left.t / \mu\left(A_{n}(x)\right)\right)$ for the distribution of the entry times to the $n$-cylinder $A_{n}(x)$. Below we will list some results when the limiting distribution is know. First however we would like to state a very general result of Downarowicz and Lacroix.

Theorem 5. 35] Let $\mu$ be an ergodic invariant measure of positive entropy and $\mathcal{A}$ a finite generating partition. Then

$$
\sup _{t \in \mathbb{R}}\left(e^{-t}-F_{x}^{n}(t)\right) \rightarrow 0
$$

in $\mathscr{L}^{1}(\Omega)$ as $n \rightarrow \infty$.

If the limiting distribution $F_{x}(t)=\lim _{n \rightarrow \infty} F_{x}^{n}(t)$ exists, then this theorem states in particular that $F_{x}(t) \geq e^{-t}$ almost surely.

2.5. The induced map. For a subset $U \subset \Omega, \mu(U)>0$, let us denote by $\hat{T}=T^{\tau_{U}}: U \rightarrow$ $U$ the induced map. By Poincaré's (or Kac's) theorem $\hat{T}$ exists almost everywhere. We also have the induced measure $\hat{\mu}$ which is defined on $U$ by $\hat{\mu}(A)=\frac{\mu(A)}{\mu(U)}$ for all measurable $A \subset U$. Recall that $\hat{\mu}$ is $\hat{T}$-invariant and also that $\hat{\mu}$ is ergodic if $\mu$ is ergodic and vice versa under the condition that $\Omega \subset \bigcup_{j} T^{j} U$.

The following theorem from 2011 shows that a restricted system $(U, \hat{T}, \hat{\mu})$ has the same limiting entry times distribution as the original system $(\Omega, T, \mu)$.

Theorem 6. 44] Let $\mu$ be ergodic, $U \subset \Omega, \mu(U)>0$. Assume there exists a sequence of sets $B_{n} \subset U, \mu\left(B_{n}\right) \rightarrow 0^{+}$, so that

$$
\begin{array}{lll}
F(t)=\lim _{n \rightarrow \infty} F_{B_{n}}(t), & F_{B_{n}}(t)=\mathbb{P}\left(\tau_{B_{n}}>\frac{t}{\mu\left(B_{n}\right)}\right) \\
\hat{F}(t)=\lim _{n \rightarrow \infty} \hat{F}_{B_{n}}(t), & \hat{F}_{B_{n}}(t)=\mathbb{P}\left(\hat{\tau}_{B_{n}}>\frac{t}{\hat{\mu}\left(B_{n}\right)}\right)
\end{array}
$$

where

$$
\tau_{B}(x)>\min \left\{j \geq 1: T^{j} x \in B\right\}, \quad \hat{\tau}_{B}(x)>\min \left\{j \geq 1: \hat{T}^{j} x \in B\right\}
$$

and $\hat{T}=T^{\tau_{U}}$ is the induced transformation on $U$.

Then $F(t)=\hat{F}(t)$ for all $t \in \mathbb{R}^{+}$. 
The same result holds for the return times distributions $\tilde{F}$ and its counterpart for the induced map $\tilde{\hat{F}}$. In fact this result was for ergodic Radon measures $\mu$ proven in [17] in 2003 where the Lebesgue Density theorem was used and the limit was along metric balls $B_{n}$ that shrink to a point $x \in \Omega$.

In conjunction with Theorem 4, we see that the limiting return times distribution of the restricted system $(U, \hat{T}, \hat{\mu})$ (for some positive measure $U \subset \Omega$ ) is the same as the limiting return times distribution of the entire system $(\Omega, T, \mu)$.

\section{Mixing properties}

In the previous sections we considered ergodic measures and obtained some general properties of the entry and return times distributions. If we wish to prove more specific results then we have to make some assumptions on mixing properties of the measure.

Let $\mathcal{A}$ be a (possibly countably infinite) measurable partition of $\Omega$ and denote by $\mathcal{A}^{n}=\bigvee_{j=0}^{n-1} T^{-j} \mathcal{A}$ its $n$-th join which also is a measurable partition of $\Omega$ for every $n \geq 1$. The atoms of $\mathcal{A}^{n}$ are called $n$-cylinders. Let us put $\mathcal{A}^{*}=\bigcup_{n=1}^{\infty} \mathcal{A}^{n}$ for the collection of all cylinders in $\Omega$ and put $|A|$ for the length of a cylinder $A \in \mathcal{A}^{*}$, i.e. $|A|=n$ if $A \in \mathcal{A}^{n}$.

We shall assume that $\mathcal{A}$ is generating, i.e. that the atoms of $\mathcal{A}^{\infty}$ are single points in $\Omega$.

3.1. Various kinds of mixing. The following is a list of mixing properties arranged in decreasing order of strength. Except for the $\beta$-mixing case, $U$ is always in the $\sigma$-algebra generated by $\mathcal{A}^{n}$ and $V$ lies in the $\sigma$-algebra generated by $\mathcal{A}^{*}$ (see also [33, 16]). The limiting behaviour is as the length of the 'gap' $\Delta \rightarrow \infty$ :

(1) $\psi$-mixing: $\sup _{n} \sup _{U, V}\left|\frac{\mu\left(U \cap T^{-\Delta-n} V\right)}{\mu(U) \mu(V)}-1\right|=\psi(\Delta) \rightarrow 0$.

(2) Left $\phi$-mixing: $\sup _{n} \sup _{U, V}\left|\frac{\mu\left(U \cap T^{-\Delta-n} V\right)}{\mu(U)}-\mu(V)\right|=\phi(\Delta) \rightarrow 0$.

$$
\beta \text {-mixing: } \sup _{n, m} \sum_{B \in \mathcal{A}^{n}, C \in T^{-n-\Delta} \mathcal{A}^{m}}|\mu(B \cap C)-\mu(B) \mu(C)| \rightarrow 0
$$

(4) Strong mixing [69] (or $\alpha$-mixing): $\sup _{n} \sup _{U, V}\left|\mu\left(U \cap T^{-\Delta-n} V\right)-\mu(U) \mu(V)\right|=\alpha(\Delta) \rightarrow$ 0 .

(5) Uniform mixing [69, [70]: $\sup _{n} \sup _{U, V}\left|\frac{1}{k} \sum_{j=1}^{k} \mu\left(U \cap T^{-n-j} V\right)-\mu(U) \mu(V)\right| \rightarrow 0$ as $k \rightarrow$ $\infty$

One can also have right $\phi$-mixing when $\sup _{n} \sup _{U, V}\left|\frac{\mu\left(U \cap T^{-\Delta-n} V\right)}{\mu(V)}-\mu(U)\right| \rightarrow 0$ as $\Delta \rightarrow \infty$. The strongest mixing property is $\psi$-mixing and it implies all the other kinds of mixing. Bernoulli measures are $\psi$-mixing and so are equilibrium states on Axiom A systems for Hölder continuous potentials. The next strongest mixing property is $\phi$-mixing, then comes $\beta$-mixing. The uniform mixing property is the weakest.

\section{First RETURN TIMES}

The first result in dynamics was by Hirata [50, 51] in 1993 and for higher order return times by Pitskel [66] in 1991. The argument of Hirata uses the exponential entry times 
distribution using the Laplace transform which technically requires a fine analysis of the spectrum of the transfer operator on the shift space as one restricts to the complement of a cylinder set.

4.1. Galves-Schmitt method. In 1997 Galves and Schmitt [37] used a practical approach that uses the mixing property to get the limiting distribution or entry times for $\psi$-mixing measures.

Theorem 7. 37] Let $\mu$ be a $\psi$ mixing measure on a subshift such that $\psi$ is summable. Then there exist constants $C>1, c_{1}$ and $\alpha>0$ such that

$$
\left|\mathbb{P}\left(\tau_{A} \geq \frac{t}{\mu(A) \lambda_{A}}\right)-e^{-t}\right| \leq c_{1} \mu(A)^{\alpha}
$$

for some numbers $\lambda_{A} \in\left(C^{-1}, C\right)$ and for all cylinders $A$.

The proof involved to subdivide the time interval $[0, N]$ into smaller portions and then use the fact that $\mathbb{P}\left(\tau_{A}>s / \mu(A)\right)=1-s+o(s)$ for very small values of $s$ which are chosen to be $s=\mu(A)^{\beta}$ for some $\beta \in(0,1)$. The $\psi$-mixing property then is used to show that $\mathbb{P}\left(\tau_{A}>N\right)$ is approximately equal to $\mathbb{P}\left(\tau_{A}>s / \mu(A)\right)^{r}$ where $r=N /(s / \mu(A))$ is the number of small intervals needed. The 'gaps' that are opened up in order to use the mixing property are negligible compared to the size of the small intervals whose lengths are $s / \mu(A)$. Thus one obtains the exponential distribution in the limit when $\mu(A) \rightarrow 0^{+}$. The drawback of this approach is that it cannot be used for higher order returns because one cannot control the earlier returns and still only use the mixing property. However, Abadi [2, 1, 4, 3] extended this method to $\phi$-mixing and even $\alpha$-mixing measures and in those cases also obtained error terms.

Theorem 8. [4] (2006) Let $\mu$ be an $\alpha$-mixing measure. Suppose that $\alpha(x) x^{-\kappa}$ ( $\kappa>$ $(1+\sqrt{5}) / 2$. Then there exists a function $\lambda: \bigcup_{n} \mathcal{A}^{n} \rightarrow(0,3]$ such that for all $A \in \mathcal{A}^{n}$

$$
\lim _{n \rightarrow \infty} \sup _{t \geq 0}\left|\mathbb{P}\left(\tau_{A}>\frac{t}{\lambda(A) \mu(A)}\right)-e^{-t}\right|=0
$$

Moreover the rate of convergence of the above limit is bounded from above by $e_{h}(A)=$ $C_{0} \inf _{n \leq g \leq f \leq 1 / \mu(A)}\left(f \mu(A)+\frac{g \mu(A)+\alpha(g)}{f \mu(A)} s\right)$, where $s=\alpha^{-1}(\mu(A)+n)$ and $C_{0}$ is a positive constant.

A similar result for $\phi$-mixing measures was obtained in [10] and for rational maps with critical points and equilibrium states that have a 'supremum gap' it was done in [42.

For more specific systems a number of results were shown in [17] for the first return times. These are one-dimensional real and complex systems that have exponential decay of correlations. Here the return times are for metric balls which in the case of the complex maps requires a regularising approximation of the characteristic function for the balls by unions of cylinder sets. For interval maps that have critical points of parabolic fixed points the exponential limiting distribution is shown for an induced map which is uniformly expanding and then by Theorem 6 translated to the original map.

As it appears that all known systems with positive entropy have exponentially distributed limiting entry/return times, Kupsa [59] has recently constructed an example of a 
positive entropy system on a three element subshift whose limiting entry/return times are not exponentially distributed in the limit. Also, Downarowics, Lacroix and Leandri [36] have constructed examples where the limiting entry time exists along subsequences of upper density one and can be arbitrarily slowly decaying to zero. Interestingly enough, it is still an open question whether the limiting entry times distribution $F(t)$ decays to zero if the limit $F(t)=\lim _{n} F_{n}(t)$ exists, i.e. whether the sequence $F_{n}$ is tight. In the mentioned examples, the limit does not seem to exist.

Recently Freitas, Freitas and Todd have established a relation between extremal values laws and entry times distribution first for absolutely continuous measures in [39] and then for general invariant measures in [40].

\section{Higher order RETURN TIMES}

Let $A \subset \Omega$ and $t>0$ a parameter, then we put $\zeta_{A}^{t}=\sum_{j=1}^{[t / \mu(A)]} \chi_{A} \circ T^{j}$ for the counting function that counts the number of times the orbit of a point $x$ visits the set $A$ on the orbit segment of length $\frac{t}{\mu(A)}$. Clearly $\zeta_{A}^{t}(x)=0$ exactly if $\tau_{A}(x)>\frac{t}{\mu(A)}$. If the return times $\tau_{A} \circ \hat{T}$ are independent $\left(\hat{T}\right.$ is the induced map on $A$ ) and are exponentially distributed then $\zeta_{A}^{t}$ will be Poisson distributed. However in a deterministic system we only get independence of entries only in the limit as their separation goes to infinity.

The first result on higher returns was due to Doeblin in 1940 for the Gauss map at the origin. Then there was a long gap and nothing much seems to have happened until 1991 when several people simultaneously began to work in this area with different methods. To recall Doeblin's result on the Gauss map let $\Omega=(0,1]$ be the unit interval. The Gauss map is then given by $T x=\frac{1}{x} \bmod 1$ and is related to the continued fraction expansion of real numbers. If $\left[a_{0}, a_{1}, a_{2}, \ldots\right]$ is the continued fraction expansion of a point $x \in \Omega$ then

$$
x=\frac{1}{a_{0}+\frac{1}{a_{1}+\frac{1}{a_{2}+\cdots}}}
$$

where the integers $a_{j} \in \mathbb{N}$ are uniquely determined by $x$ and are given by $a_{j}=\frac{1}{x}-T x$. The Gauss measure $\mu$ on $(0,1]$ is the unique absolutely continuous $T$-invariant probability measure. Its density is $\frac{1}{\log 2} \frac{1}{1+x}$ and Doeblin [31] showed that for every $\theta>0$ :

$$
\mathbb{P}\left(\left|\left\{j: 1 \leq j \leq n, a_{j}(x) \geq \theta n\right\}\right|=p\right) \rightarrow e^{-1 /(\theta \log 2)} \frac{1}{(\theta \log 2)^{p} p !}
$$

as $n \rightarrow \infty$. Since $x=\frac{1}{a_{j}+T x}=\frac{1}{a_{j}+\mathcal{O}(1)}($ as $0<T x \leq 1)$ we see that a point $x \in(0,1]$ for which $\left|\left\{j: 1 \leq j \leq n, a_{j}(x) \geq \theta n\right\}\right|=p$ visits the interval $\left(0, \frac{1}{n \theta}\right)$ typically exactly $p$ times on the orbit segment of length $n$. Since $\mu\left(\left(0, \frac{1}{n \theta}\right)\right)=\frac{1}{\log 2} \log \left(1+\frac{1}{\theta n}\right) \approx \frac{1}{\log 2} \frac{1}{\theta n}$ we can put $A_{m}=\left(0, \frac{1}{m}\right)$ and see that Doeblin's statement translates into

$$
\mathbb{P}\left(\zeta_{A_{m}}^{t}=p\right) \rightarrow e^{-t} \frac{t^{p}}{p !}
$$

as $m \rightarrow \infty$. In other words, the limiting distribution of return times at the origin is Poissonian. 
There are several ways in which limiting results on higher order returns have been achieved. Here we will mention results that use the moment method, the Chen-Stein method, direct estimates of the total variation norm and a combinatorial approach.

5.1. Moment method. The first dynamical result dates to 1991 and is due to Pitskel [66]. It uses the method of moments which was also used by Wang, Tang and Wang [75] and Denker [29] for Gibbs measures on shift spaces. For a set $A \subset \Omega$ and a parameter $t>0$ let us put

$$
\zeta_{A}^{t}=\sum_{j=1}^{N} \chi_{A} \circ T^{j}
$$

for the counting function on the orbit segment of length $N=\left[\frac{t}{\mu(A)}\right]$. The value $\zeta_{A}^{t}(x)$ counts the number of times the orbit of $x \in \Omega$ enters $A$ up to time $N$. Now let

$$
G_{r}=\left\{\left(v_{1}, v_{2}, \ldots, v_{r}\right): 1 \leq v_{1}<v_{2}<\cdots<v_{r} \leq N\right\}
$$

be the set of possible $r$-fold return times of a point to a given set within the time interval $[1, N]$. The moment generating function for $\zeta$ is then given by

$$
\varphi(z)=\sum_{k=0}^{\infty} z^{k} \mathbb{P}(\zeta=k) .
$$

A computation verifies that

$$
\varphi(z)=\sum_{r=0}^{\infty} \frac{(z-1)^{r}}{r !} \mu\left(\xi_{r}\right),
$$

where $\xi_{r}=\zeta(\zeta-1)(\zeta-2) \cdots(\zeta-r+1)$ is the $r$-factorial moment of $\zeta$. It then follows that

$$
\frac{1}{r !} \mu\left(\xi_{r}\right)=\sum_{\vec{v} \in G_{r}} \mu\left(\bigcap_{j=1}^{r} T^{-v_{j}} A\right) .
$$

If $\zeta$ is Poisson distributed with parameter $t>0$, then $\mathbb{P}(\zeta=k)=e^{-t} \frac{t^{k}}{k !}$, the generating function is $e^{t(z-1)}$ and one has $\mu\left(\xi_{r}\right)=t^{r}$. The advantage of this approach lies in the fact that on the right hand side of this identity one can use the mixing property, as it involves the intersection of pull backs of $A$. One opens up 'gaps' and uses rough estimates on short returns and shows that their cardinality is 'small'. Most of the 'return time patterns' $\vec{v}$ are however long and there one can use the mixing property. Because one has to look at arbitrarily high orders $r$ of mixing, this approach limits itself to $\psi$-mixing measures like Axiom A maps.

Now according to a theorem of Sevast'yanov [73] if the moments $\mu\left(\xi_{r}\right)$ for $A=A_{n}(x)$ converge to $t^{r}$ as $n \rightarrow \infty$ then $\zeta_{A_{n}(x)}^{t}$ converges in distribution to a Poisson distribution with parameter $t$. In this way Pitskel proved the following result in 1991:

Theorem 9. 66] Let $\mu$ be an equilibrium state for a Hölder continuous potential on a subshift of finite type. Then for all $t>0$

$$
\mathbb{P}\left(\zeta_{A_{n}(x)}^{t}=r\right) \rightarrow \frac{t^{r}}{r !} e^{-t}
$$


as $n \rightarrow \infty$ almost surely in $x \in \Omega$.

He then used an approximation argument to extend this result to metric balls for twodimensional toral automorphisms.

This approach was also used in 43 to prove that the limiting return times distributions are Poissonian for equilibrium states of rational maps. There an approximation argument was used to show that the return times to metric balls are in the limit Poisson distributed. In 2003 we went further and obtained that for a somewhat more general class of $\psi$-mixing maps one has Poisson distributed entry and return times in the limit [48]. An improved moment method also gave error terms which depend on the rate at which $\psi(k)$ decreases to zero as $k$ goes to infinity. The key to this is to obtain a Sevast'yanov type theorem that allows error estimates and to show that approximations of the moments translate into an approximation of the distribution to the Poisson distribution.

5.2. Laplace transform. This is the method that was first used by Hirata [50] in 1993 for equilibrium states on subshifts of finite type. He proved that the first entry time is exponentially distributed in the limit and then argued that the weak mixing property implies that higher order returns are in the limit Poisson distributed. This requires a careful analysis of the transfer operator acting on the complement of the cylinder target set and delicate estimates on the dominant eigenvalue $e^{P(f)}$ where $P(f)$ is the pressure of the potential function $f$ (see e.g. [15]). This approach was more conclusively carried out by Coelho and Collet [24] and also in [20] for measures on subshifts that have strong mixing properties. The combinatorics involved tend to make such an approach difficult and would favour the moment method.

5.3. Chen-Stein method. Let $\nu$ be a probability measure on $\mathbb{N}_{0}$ and denote by $\nu_{0}$ the Poisson-distribution measure with mean $t$, i.e. $\mathbb{P}_{\nu_{0}}(\{k\})=\frac{e^{-t} t^{k}}{k !} \forall k \in \mathbb{N}_{0}$. If we put $\mathcal{F}$ for the set of all real-valued functions on $\mathbb{N}_{0}$, then the Stein operator $\mathcal{S}: \mathcal{F} \rightarrow \mathcal{F}$ is defined by

$$
\mathcal{S} f(k)=t f(k+1)-k f(k), \quad \forall k \in \mathbb{N}_{0},
$$

where $f \in \mathcal{F}$. The Stein equation, given by

$$
\mathcal{S} f=h-\int_{\mathbb{N}_{0}} h d \nu_{0}
$$

then has for every $\nu_{0}$-integrable $h \in \mathcal{F}$ a solution $f$ which is unique except for $f(0)$ which can be chosen arbitrarily [13]1. A probability measure $\nu$ on $\mathbb{N}_{0}$ is Poisson (with parameter $t$ ) if and only if $\int_{\mathbb{N}_{0}} \mathcal{S} f d \nu=0$ for all bounded functions $f \in \mathcal{F}$.

If $E \subset \mathbb{N}_{0}$ then one can take $h=\chi_{E}$ the characteristic function and estimate the distance of a probability measure $\nu$ on $\mathbb{N}_{0}$ from the Poisson distribution $\nu_{0}$ as follows:

$$
\left|\nu(E)-\nu_{0}(E)\right|=\left|\int_{\mathbb{N}_{0}} \mathcal{S} f d \mu\right|=\left|\int_{\mathbb{N}_{0}}(t f(k+1)-k f(k)) d \mu\right|=\left|\mathbb{P}(\zeta \in E)-\mu_{0}(E)\right|
$$

${ }^{1}$ In fact $f$ can be computed recursively:

$$
f(k)=\frac{(k-1) !}{t^{k}} \sum_{i=0}^{k-1}\left(h(i)-\nu_{0}(h)\right) \frac{t^{i}}{i !}=-\frac{(k-1) !}{t^{k}} \sum_{i=k}^{\infty}\left(h(i)-\nu_{0}(h)\right) \frac{t^{i}}{i !}, \quad \forall k \in \mathbb{N}
$$


where $f$ is a solution to the Stein equation. An estimate on the function $f$ then allows us to bound the distance between $\nu$ and $\nu_{0}$ using mixing properties of up to second order.

5.3.1. Toral automorphisms. In 2004 Denker, Gordin and Sharova [30] applied this method to the Haar measure of hyperbolic toral automorphism to show that the limiting return times distribution is Poissonian in the limit if one considers successive returns ball-like sets $G_{n}$ which contract to a non-periodic point. The return sets $G_{n}$ lie inside balls and have a measure that is comparable. Interestingly enough, this result shows a dichotomy that at non-periodic points one always gets the limit Poisson distributed return times.

5.3.2. Mixing measures. Abadi used in his thesis in 2001 and in [5] a theorem of Arratia, Goldstein and Gordon [12] to get the Poisson limiting distribution for $\phi$-mixing systems. Exploiting the fact that with the Chen-Stein method only two-fold mixing is required in order to get the Poisson distribution, it was shown that

$$
\mathbb{P}\left(\zeta_{A_{n}}^{t}(x)=k\right)=e^{-t} \frac{t^{k}}{k !}+\mathcal{O}(f(t) \epsilon(n))
$$

for an error term function $f$ which depends on the parameter $t$ and $\epsilon$ depending on the length $n$ of the "target string" and the point $x \in \Omega$. Different error term functions $\epsilon$ are given for $\psi$-mixing measures and $\beta$-mixing measures. In 2008 these results were improved by Abadi and Vergne [9] to $\phi$-mixing and $\alpha$-mixing systems respectively using a different approach. Their improved result uses a more elementary method combined with the exponential entry time results of Abadi's earlier papers. All thse results hold if restricted to returns only in which case one uses the induced measure on the initial set $A_{n}(\cdot)$ although the error terms are slightly different.

This extends a previous result of Hirata, Saussol and Vaienti [52] where error estimates for $\mathbb{P}\left(\zeta_{U}^{t}\right)-e^{-t} \frac{t^{r}}{r !}$ are given under the assumption that the partition $\left\{U, U^{c}\right\}$ is $\phi$-mixing or $\alpha$-mixing (different error terms). This result was then used to obtain the Poisson distribution and rates of convergence for higher order limiting return times for parabolic interval maps.

Using the Chen-Stein method, Psiloyenis proved in 2008 a similar result which allows the return set to be a possibly infinite unions of cylinder sets. The alphabet in this case can be countably infinite and the entropy is not required to be finite.

Theorem 10. 68, 45] Let $\mu$ be a $\phi$-mixing T-invariant probability measure with an at most countably infinite partition $\mathcal{A}$. Let $A \in \sigma\left(\mathcal{A}^{n}\right)$ be a finite or infinite union of $n$ cylinders such that $|\log \mu(A)|=\mathcal{O}\left(n^{\eta}\right)$ and $n^{\eta} \phi(n) \rightarrow 0$ as $n \rightarrow \infty$ for some $\eta \geq 1$ and $r_{A}=\mathcal{O}(n)$. Then for all $t>0$ :

(i) Exponential mixing rate: $\left(\phi(k)=\mathcal{O}\left(\vartheta^{k}\right)\right.$, with $\left.\vartheta \in(0,1)\right)$ there exists $\gamma=\gamma(\vartheta)>$ 0

$$
\mathbb{P}\left(\zeta_{A}^{t}=r\right)=e^{-t} \frac{t^{r}}{r !}+\mathcal{O}(t(t \vee 1)) e^{-\gamma n} .
$$

(ii) Polynomial mixing rate: $\left(\phi(k)=\mathcal{O}\left(k^{-\beta}\right)\right.$ with $\left.\beta>1+\eta\right)$,

$$
\mathbb{P}\left(\zeta_{A}^{t}=r\right)=e^{-t} \frac{t^{r}}{r !}+\mathcal{O}(t(t \vee 1)) n^{-(\beta-1-\eta)}
$$


Here $r_{A}$ denotes the first return of $A$ to itself, i.e. $r_{A}=\inf _{x \in A} \tau_{A}(x)$ and the lower bound is required to control short returns and to exclude 'periodic like' behaviour. The lower cutoff for $r_{A}$ is not optimal but is a typical requirement to exclude 'periodic looking' target sets $A$.

Recently Kifer [55] used a similar method to obtain results on the limiting distribution of simultaneous returns. Following the approach taken by Abadi using a theorem of Arratia, Goldstein and Gordon [12], Kifer showed that simultaneous returns are in the limit Poissonian. For $\ell$ simultaneous returns one lets $q_{1}(j)<q_{2}(j)<\cdots<q_{\ell}(j)$ be the return times so that $g(j)=\min _{k}\left(q_{k}(j)-q_{k-1}(j)\right)$ goes to infinity as $j \rightarrow \infty$. Then one forms the counting function for simultaneous hits at times $q_{k}$ by putting

$$
\xi_{A}^{t}=\sum_{j=1}^{\left[t / \mu(A)^{\ell}\right]} \prod_{k=1}^{\ell} \chi_{A} \circ T^{q_{k}(j)}
$$

for $x \in \Omega$. For $\ell=1$ and $q_{1}(j)=j$ this reduces to the standard case considered above.

Theorem 11. [55] If $\mu$ is $\psi$-mixing, then for $A \in \mathcal{A}^{n}$ :

$$
\mathbb{P}\left(\xi_{A}^{t}=r\right)=e^{-t} \frac{t^{r}}{r !}+\mathcal{E}(A) .
$$

The error term $\mathcal{E}(A)$ is up to polynomial terms in $\ell, n$ (and depending on $g$ ) and exponential terms in $t$ equal to $\mu(\tilde{A})+\psi(n)$, where $\tilde{A}=A_{r_{A}^{\prime}}(A)$ is the $r_{A}^{\prime}$-cylinder containing $A$ where $r_{A}^{\prime}=\min \left(\inf \left\{j \geq 0: T^{j} A \cap A \neq \emptyset\right\}, n\right)$.

In [54] this result was proven without error terms.

5.3.3. Markov towers. L-S Young's construction of Markov towers has proven to be a very powerful tool to obtain results on the statistical properties of maps and the method can also be used to obtain results on the return times distribution. Let us recall the construction from [77, 78].

For a differentiable map $F$ on a manifold $M$ one lets $\Omega_{0}$ be a subset of $M$ which is partitioned into sets $\Omega_{0, i}, i=1,2, \ldots$ so that there is a return time function $R$ : $\Omega_{0} \rightarrow \mathbb{N}$ which is constant on the partition elements $\Omega_{0, i}$ and which satisfies that $F^{R}$ maps $\Omega_{0, i}$ bijectively to the entire set $\Omega_{0}$. If we put $\Omega_{j, i}=\left\{(x, j): x \in \Omega_{0, i}\right\}$ for $j=$ $0,1, \ldots, R\left(\Omega_{0, i}\right)-1$ then $\Omega=\bigcup_{i=1}^{\infty} \bigcup_{j=0}^{R\left(\Omega_{0, i}\right)-1} \Omega_{j, i}$ is called a Markov tower for the map $T$ given by

$$
T(x, j)=\left\{\begin{array}{ll}
(x, j+1) & \text { if } j<R(x)-1 \\
\left(F^{R}(x), 0\right) & \text { if } j=R(x)-1
\end{array} .\right.
$$

It has the (typically) countably infinite partition $\mathcal{A}=\left\{\Omega_{j, i}: i, j\right\}$.

The separation function $s(x, y)$ is the smallest positive $n$ so that $\left(T^{R}\right)^{n} x$ and $\left(T^{R}\right)^{n} y$ lie in distinct sub-partition elements $\Omega_{0, i}$ of $\Omega_{0}$. Two points $x$ and $y$ in $\Omega$ belong to the same $N$-cylinder if and only if they remain together (in the same partition element) for at least $n$ iterations of $T^{R}$, i.e. if $s(x, y) \geq n$, where $N=\sum_{j=0}^{n-1} R\left(T^{R}\right)^{j}$.

The space of Hölder continuous functions $\mathcal{C}_{\gamma}$ consists of all functions $\varphi$ on $\Omega$ for which $|\varphi(x)-\varphi(y)| \leq C_{\varphi} \gamma^{s(x, y)}$. The norm on $\mathcal{C}_{\gamma}$ is $\|\varphi\|_{\gamma}=|\varphi|_{\infty}+C_{\varphi}$, where $C_{\varphi}$ is smallest possible. 
Let $\nu$ be a finite given 'reference' measure on $\Omega$ and assume that the Jacobian $J T^{R}$ with respect to the measure $\nu$ is Hölder continuous, that is, there exists a $\gamma \in(0,1)$ so that

$$
\left|\frac{J T^{R} x}{J T^{R} y}-1\right| \leq \operatorname{const}^{s\left(T^{R} x, T^{R} y\right)}
$$

for all $x, y \in \Omega_{0, i}, i=1,2, \ldots$.

If the return time $R$ is integrable with respect to $m$ then by [78] Theorem 1 there exists a $T$-invariant probability measure $\mu$ (SRB measure) on $\Omega$ which is absolutely continuous with respect to $\nu$. Moreover the density function $h=\frac{d \mu}{d \nu}=\lim _{n \rightarrow \infty} \mathcal{L}^{n} \lambda$ is Hölder continuous, where $\lambda$ can be any initial density distribution in $\mathcal{C}_{\gamma}$. The transfer operator $\mathcal{L}: \mathcal{C}_{\gamma} \rightarrow \mathcal{C}_{\gamma}$ is defined by $\mathcal{L} \varphi(x)=\sum_{x^{\prime} \in T^{-1} x} \frac{\varphi\left(x^{\prime}\right)}{J T\left(x^{\prime}\right)}, \varphi \in \mathcal{C}_{\gamma}$, and has the property that $\nu$ is a fix point of its adjoint, i.e. $\mathcal{L}^{*} \nu=\nu$. In [78] Theorem 2(II) the $L^{1}$-convergence was proven:

$$
\left\|\mathcal{L}^{k} \lambda-h\right\|_{L^{1}} \leq p(k)\|\lambda\|_{\gamma}
$$

where the 'decay function' $p(k)=\mathcal{O}\left(k^{-\beta}\right)$ if the tail decays polynomially with power $\beta$, that is if $\nu(R>j) \leq$ const. $j^{-\beta}$. If the return times decay exponentially, i.e. if $\nu(R>j) \leq$ const. $\vartheta^{j}$ for some $\vartheta \in(0,1)$, then there is a $\tilde{\vartheta} \in(0,1)$ so that $p(k) \leq$ const. $\tilde{\vartheta}^{k}$.

Theorem 12. [45] As described above let $T$ be a map on the Markov Tower structure $\Omega$ with a reference measure $\nu$ and a return time function $R$. Let $\mu$ be the absolutely continuous invariant measure. Let $K$ be a constant. Then for every $A_{n} \in \sigma\left(\mathcal{A}^{n}\right)$ for which $\left|\log \mu\left(A_{n}\right)\right| \leq K n, r_{A_{n}}>\frac{n}{2}$ and $\sup _{A_{n}} R \leq \frac{n}{2}$, the following result holds true:

If $\nu(R>n)=\mathcal{O}\left(\vartheta^{n}\right)(\vartheta \in(0,1))$ or if $\nu(R>n)=\mathcal{O}\left(n^{-\beta}\right)$ for some $\beta>2$, then there exists $\gamma>0$ such that

$$
\mathbb{P}\left(\zeta_{A_{n}}^{t}=r\right)=e^{-t} \frac{t^{r}}{r !}+\mathcal{O}\left((t \vee 1) e^{-\gamma n}\right) \quad \forall t>0 \text { and } \forall n \in \mathbb{N} .
$$

5.4. Total variation estimates. In 2010 Chazottes and Collet [21] proved the Poisson distribution for Young's Markov towers in the codimension one case when the tails decay at an exponential rate, i.e. $\nu(R>n) \sim \vartheta^{n}$ for a $\vartheta \in(0,1)$. The estimates use the decay of correlation and require that the characteristic functions of the metric balls be approximated by Lipschitz continuous functions. This introduces additional difficulties as it is necessary to control the contribution made by an annulus surrounding the metric balls on which the approximating function interpolates between the values 0 and 1 .

Theorem 13. 21] Let $(M, T, \mu)$ be a non-uniformly hyperbolic dynamical system modelled by a Young tower whose return-time function has an exponential tail. Assume that the local unstable manifolds have dimension one. Denote by $\mu$ its SRB measure.

Then there exist constants $a, b>0$ such that for all $\rho \in(0,1)$ :

(i) There exists a set $M_{\rho}$ such that $\mu\left(M_{\rho}\right)=\mathcal{O}\left(\rho^{b}\right)$;

(ii) For all $x \notin M_{\rho}$ one has $\left(B_{\rho}(x)\right.$ is the metric ball with centre $x$ and radius $\rho$ )

$$
\mathbb{P}\left(\sum_{j=0}^{N} \chi_{B_{\rho}(x)}=r\right)=e^{-t} \frac{t^{r}}{r !}+\mathcal{O}\left(\rho^{a}\right)
$$

for all $\rho \geq 0$ small enough and for every $t>0$. 
A similar result for polynomially decaying correlations has now been proven by Wasilewska [76] with error terms which are polynomially decaying in $|\log \rho|$.

\section{Periodic orbits}

Hirata [50] and Abadi [1] have pointed out that at a periodic point the limiting return time is not exponentially distributed like $e^{-t}$ but that it is a combination of a Dirac pointmass at the origin with a rescaled exponential decay. This can easily be seen for a Bernoulli measure on a full two element shift $\Sigma$. On $\Sigma$ one has the left shift $\sigma$. If $\mu$ is the Bernoulli measure for the probabilities $p, 1-p$ for some $p \in(0,1)$, and $\vec{x}=0^{\infty}$ the fixed point whose coordinates are all 0s, then $F_{\vec{x}}^{n}(t)=\mathbb{P}_{A_{n}(\vec{x})}\left(\tau_{A_{n}(\vec{x})} \geq t / \mu\left(A_{n}(\vec{x})\right)\right)$ has the limiting distribution $F_{\vec{x}}(t)=(1-p) e^{t /(1-p)}$ for $t>0$. Equivalently one obtains that the limiting distribution of $\mathbb{P}_{A_{n}(\vec{x})}\left(\tau_{A_{n}(\vec{x})} \geq t /\left[(1-p) \mu\left(A_{n}(\vec{x})\right)\right]\right)$ is $(1-p) e^{-t}$. This is the way it is formulated in [1]. For an equilibrium states for a Hölder continuous potential $\varphi$ on a subshift of finite type this was formulated by by Hirate [50] for arbitrary periodic points where it was shown that at a periodic point $x$ with minimal period $m$

$$
\lim _{n \rightarrow \infty} \mathbb{P}_{A_{n}(x)}\left(\tau_{A_{n}(x)} \geq \frac{t}{(1-p) \mu\left(A_{n}(x)\right)}\right)=(1-p) e^{-t}
$$

where $p=e^{\sum_{j=0}^{m-1} \varphi \circ T^{j}}$.

If $\mu$ is a $\psi$-mixing measure then for a periodic point $x$ with minimal period $m$ the limit $p=\lim _{\ell \rightarrow \infty}\left|\frac{1}{\ell} \log \mu\left(A_{\ell m}(x)\right)\right|$ exists. In particular $p$ is always strictly less than 1 . In the following we shall assume the stronger property that $p=\lim _{n \rightarrow \infty} \frac{\mu\left(A_{n+m}(x)\right)}{\mu\left(A_{n}(x)\right)}$. This of course implies the limit in the lemma, but we are not sure whether the reverse implication is generally true. Also put $q_{n}=\sup _{\ell \geq n}\left|\frac{\mu\left(A_{\ell+m}(x)\right)}{\mu\left(A_{\ell}(x)\right)}-p\right|$. For $t>0$ and integers $n$ we put $\zeta_{n}^{t}$ for the classical counting function on cylinder sets $\sum_{j=0}^{N_{n}} \chi_{\left.A_{n}(x)\right)} \circ T^{j}$ with the rescaled observation time

$$
N_{n}=\left[\frac{t}{(1-p) \mu\left(A_{n}(x)\right)}\right] .
$$

The limiting distribution of $\zeta_{n}^{t}$ is the Pólya-Aeppli distribution according to which the value $r \in \mathbb{N}_{0}$ is assumed with probability $e^{-t} P_{r}(t, p)$ where

$$
P_{r}(t, p)=\sum_{j=1}^{r} p^{r-j}(1-p)^{j} \frac{t^{j}}{j !}\left(\begin{array}{c}
r-1 \\
j-1
\end{array}\right) .
$$

To be more precise we have the following result

Theorem 14. 49] Let $\mu$ be a $\psi$-mixing measure with partition $\mathcal{A}$ (finite or infinite), $x$ a periodic point with minimal period $m$ and $p$ and $q_{n}$ as above. Then

$$
\mathbb{P}\left(\zeta_{n}^{t}=r\right)=e^{-t} P_{r}+\mathcal{E}\left(A_{n}(x)\right) .
$$

The error term $\mathcal{E}\left(A_{n}(x)\right)$ is up to exponential terms in $t$ and rapidly decaying terms in $r$ roughly of the form $\inf _{\delta}\left(\delta \mu\left(A_{n}(x)\right)+\psi(\delta)\right)+p^{\frac{n}{m}}+q_{n}$. 
In the case of an infinite partition $\mathcal{A}$ no finiteness of entropy is required.

If $\mu$ is an equilibrium state for a Hölder continuous function $f$ on an Axiom A space (shift space) which has the finite, generating partition $\mathcal{A}$ (see [15]) then the error term $\mathcal{E}$ can be optimised to yield, again up to exponential terms in $t$ and rapidly decaying terms in $r$, roughly $n \mu\left(A_{n}\right)+p^{\frac{n}{m}}$. In [41] the compound Poisson distribution for extremal values distribution and by extension also for the return times distribution was proven at repelling fixed points for some non-uniformly hyperbolic systems.

Recently in 2012 Kifer proved a general result on points where the limiting distribution is not Poissonian. For $\ell$ simultaneous returns at times $q_{k}(j)=d_{k} j, k=1, \ldots, \ell$ where $1 \leq d_{1}<d_{2}<\cdots<d_{\ell}$ are integers one puts as before

$$
\xi_{A}^{t}=\sum_{j=1}^{\left[t / \mu(A)^{\ell}\right]} \prod_{k=1}^{\ell} \chi_{A} \circ T^{d_{k} j} .
$$

Let $r_{A}=\inf \left\{j \geq 0: T^{j} A \cap A \neq \emptyset\right\}$, put $\kappa_{A}=\operatorname{lcm}\left(r_{A} / \operatorname{gcd}\left(r_{A}, d_{i}\right): i\right)$ and define $\left(r=r_{A}\right)$

$$
\rho_{A}=\prod_{k=1}^{\ell} \mu_{A}\left(\bigcap_{i=0}^{\left[\left(n+d_{k} \kappa\right) / r\right]} T^{-i r} A \cap T^{-[n / r]} A_{n-r[n / r]}(A)\right)
$$

where $A_{n-r[n / r]}(A)$ denotes the unique $n-r[n / r]$-cylinder containing $A$.

Theorem 15. 55. If $\mu$ is $\psi$-mixing, then for every $A \in \mathcal{A}^{n}$ and $n$ which satisfies $n>$ $r_{A}\left(6+d_{\ell}\right)$ there are iid random variables $\eta_{1}, \eta_{2}, \ldots$ with values in $\left\{1,2, \ldots,\left[n / r_{A}\right]\right\}$ and independent of the Poissonian $Z$ with parameter $t\left(1-\rho_{A}\right)$ such that

$$
\mathbb{P}\left(\xi_{n}^{t} \in L\right)=\mathbb{P}(W \in L)+\mathcal{E}(A) \quad \forall L \subset \mathbb{N}_{0},
$$

where $W=\sum_{i=1}^{Z} \eta_{i}$ is the associated compound Poisson random variable.

The error term $\mathcal{E}(A)$ equals $e^{-\gamma n}+\psi(n)$ up to polynomial terms in $n$ and exponential terms in $\ell, t$, where $\gamma>0$ is such that $\mu(B) \leq e^{-2 \gamma m} \forall B \in \mathcal{A}^{m} \forall m \in \mathbb{N}$.

This result completely describes (subject to the condition $n>r_{A}\left(6+d_{\ell}\right)$ ) the distribution at every point, periodic or not. In the case $\ell=1$ and $d_{1}=1$ at a periodic point $x$ of minimal period $m$ we have $r=m$ for $n$ large enough, $\rho=p$ from Theorem 14 and the random variables $\eta_{i}$ can be replaced by random variables that are geometrically distributed with parameter $p$. The limiting result is then the same as in Theorem 14 although the error terms are larger.

\section{RECURRENCE TIMES}

Recurrence time is the special case when the point whose reentry is observed is the same at which the target cylinders are centered. To be precise, let $\mathcal{A}$ be a generating finite or countably infinite partition of $\Omega$, then $R_{n}(x)=\tau_{A_{n}(x)}(x)$ is the recurrence time and measures the time it takes for the first $n$ symbols of a point $x$ to reoccur in $x$. In the symbolic description, when every point $x$ is identified by its trajectory $\vec{x}=\left(\ldots, x_{-1}, x_{0}, x_{1}, \ldots\right)$ then

$$
R_{n}(x)=\min \left\{j \geq 1: x_{j} x_{j+1} \cdots x_{j+n-1}=x_{0} x_{1} \cdots x_{n-1}\right\}
$$


measures the time it takes until one sees the starting $n$-word again. According to Kac's theorem the value of $\tau_{A_{n}(x)}$ is on average $1 / \mu\left(A_{n}(x)\right)$. Denote by $h(\mu)$ the measure theoretic entropy of the invariant probability measure $\mu$. According to the theorem of Shannon-McMillan-Breiman [61] one has $\mu\left(A_{n}(x)\right) \sim e^{-n h}$ which would one make expect that $R_{n}(x) \sim e^{n h}$. This indeed is true as was proven by Ornstein and Weiss first for finite alphabets in 1993 [63] and in 2002 for countably infinite alphabets [64]:

Theorem 16. [63, 64] Let $\mu$ be ergodic and $\mathcal{A}$ a finite or countably infinite $\mu$-generating partition, then almost surely

$$
\lim _{n \rightarrow \infty} \frac{\log R_{n}(x)}{n}=h(\mu) .
$$

In the infinite case one must have $\sum_{A \in \mathcal{A}} \mu(A)|\log \mu(A)|<\infty$ in order to ensure finite entropy.

For some mixing systems this result was strengthened by Kontoyiannis [57] who prove the almost sure invariance principle. The requirement is that the invariant measure is $\alpha$ mixing of a sufficient rate $\left(\alpha(\Delta)=\mathcal{O}\left(\Delta^{-336}\right)\right)$ and satisfy an $\mathscr{L}^{1}$-Gibbs condition]2 This strengthened a previous result of Nobel and Wyner [62] who showed that for strongly mixing systems (without regularity condition) the exponential growth rate of recurrence times equals the metric entropy.

For exponentially $\psi$-mixing Gibbs measures, Collet, Galves and Schmitt [27] proved the Central Limit Theorem for the recurrence time that is $\left(\log R_{n}-n h(\mu)\right) / \sigma \sqrt{n}$ converges in distribution to the normal law (provided the variance $\sigma^{2}$, which is given by the Gibbs potential, is positive). This required the CLT for Shannon-McMillan-Breiman and the fact that entry times are exponentially distributed.

A similar result holds for metric spaces. If $T$ is a map on a metric space $\Omega$ with metric $d$, then the $n$th Bowen ball is given by $B_{\varepsilon, n}(x)=\left\{y \in \Omega: d\left(T^{j} x, T^{j} y\right)<\varepsilon, 0 \leq j<n\right\}$. With $\mathrm{F}$ Yang we have proven that for an ergodic $T$-invariant probability measure $\mu$ one has

$$
\lim _{\varepsilon \rightarrow 0} \lim _{n \rightarrow \infty} \frac{1}{n} \log R_{\varepsilon, n}(x)=h(\mu)
$$

almost everywhere, where $R_{\varepsilon, n}(x)=\tau_{B_{\varepsilon, n}(x)}(x)$ is the recurrence time to the Bowen ball (the limit in $n$ is limsup or liminf).

For geometric balls one can define $R_{\varepsilon}(x)=\tau_{B_{\varepsilon}(x)}(x)$ for the recurrence time to the geometric ball $B_{\varepsilon}(x)$. Then $\lim _{\varepsilon \rightarrow 0} \frac{\log R_{\varepsilon}(x)}{|\log \varepsilon|} \leq d(x)$ if the limits and the dimension exist and otherwise for limsup and liminf on both sides. This was shown in [14 to be true almost everywhere for invariant measures of maps on compact manifolds. Equality was proven by Saussol [72] and more generally in [71] in the case when correlation functions decay superpolynomially.

An interesting connection to the Rényi entropy function is provided by looking at the quantity

$$
Z_{n}(t)=\sum_{j=0}^{R_{n}(x)} \mu\left(A_{n}\left(T^{j} x\right)\right)^{t}
$$

\footnotetext{
${ }^{2}$ Denote by $f_{n}(\vec{x})=-\mathbb{P}\left(x_{0} \mid x_{-1} x_{-2} \cdots x_{-n}\right)$ and let $f$ be the pointwise limit of $f_{n}$ as $n \rightarrow \infty$. It is then required that $\left\|f-f_{n}\right\|_{1}=\mathcal{O}\left(n^{-48}\right)$
} 
and its limiting behaviour as $n$ goes to infinity. For the equilibrium state $\mu$ for Hölder continuous potential $\varphi$ on a subshift of finite type its exponential growth rate is

$$
\lim _{n \rightarrow \infty} \frac{1}{n} \log Z_{n}(t)=h(\mu)+P((1+t) \varphi)-(1+t) P(\varphi)
$$

almost everywhere, where $P$ denotes the pressure function. For $t=1$ this was shown by Deschamps, Schmitt, Urbanski and Zdunik [32] by using large deviations which in this case are exponential. Evidently, for $t=0$ one recovers the statement of Ornstein and Weiss' theorem. The general case for $\phi$-mixing measures was dealt with by Ko [56] who showed that

$$
\lim _{n \rightarrow \infty} \frac{1}{n} \log Z_{n}(t)=h(\mu)-t R(t)
$$

where $R(t)=\lim _{n \rightarrow \infty} \frac{1}{n t} \log \sum_{A \in \mathcal{A}} \mu(A)^{1+t}$ is the Rényi entropy function $(R(0)=h(\mu))$.

For the generalised recurrence time we have [38]

$$
\liminf _{n \rightarrow \infty} \frac{1}{n} \log \tau_{A_{n}(z)}(x) \geq h(\mu)
$$

for $\mu \times \mu$ almost all $(z, x) \in \Omega \times \Omega$ for all ergodic invariant measures $\mu$. Unfortunately a general result as in Ornstein and Weiss' theorem cannot hold true as Shields [74] has produced an example of an invariant measure over a four element shift space for which $\limsup _{n \rightarrow \infty} \frac{1}{n} \log \tau_{A_{n}(z)}(x)=\infty$ almost surely with respect to the product measure. For Gibbs measures on subshifts of finite type it Chazottes and Ugalde [22] have proven that $\frac{1}{n} \log \tau_{A_{n}(z)}(x) \rightarrow h(\mu)$ almost surely in $\mu \times \mu$. More generally for $\phi$-mixing measures it was shown in [38] that under the assumption that the limiting entry times function $F_{x}(t)=\lim _{n \rightarrow \infty} F_{x}^{n}(t)$ exists almost surely and decays to 0 as $t$ goes to infinity, then

$$
\frac{1}{n} \log \tau_{A_{n}(z)}(x) \longrightarrow h(\mu)
$$

in measure.

\section{REFERENCES}

[1] M Abadi: Exponential Approximation for Hitting Times in Mixing Stochastic Processes; Mathematical Physics Electronic Journal 7 (2001).

[2] M Abadi: Instantes de ocorrência de eventos raros em processos misturadores; PhD thesis 2001, Universidade de São Paulo.

[3] M Abadi: Sharp error terms and necessary conditions for exponential hitting times in mixing processes; Ann. Prob. 32 (2004), 243-264.

[4] M Abadi: Hitting, returning and the short correlation function; Bull. Braz. Math. Soc. 37(4) (2006), $1-17$.

[5] M Abadi: Poisson approximations via Chen-Stein for non-Markov processes; In and Out of Equilibrium 2 V Sidoravicius and M E Vares (editors), 2008, pp1-19.

[6] M Abadi and A Galves: Inequalities for the occurrence times of rare events in mixing processes. The state of the art; Markov Proc. Relat. Fields 7 (2001) 97-112.

[7] M Abadi and A Galves: A version of Maurer's conjecture for stationary $\psi$-mixing processes; Nonlinearity 17 (2004), 1357-1366.

[8] M Abadi and B Saussol: Hitting and returning into rare events for all alpha-mixing processes; Stoch. Proc. Appl. 121 (2011), 314-323.

[9] M Abadi and N Vergne: Sharp errors for point-wise Poisson approximations in mixing processes; Nonlinearity 21 (2008), 2871-2885. 
[10] M Abadi and N Vergne: Sharp error terms for return time statistics under mixing conditions Technical; Report Imecc-Unicamp 2006.

[11] M Abadi and N Vergne: Poisson approximation for search of rare words in DNA sequences; ALEALat. Am. J. Prob. Math. Stat. 4 233-44.

[12] R Arratia, L Goldstein and L Gordon: Poisson approximation and the Chen-Stein method. With comments and a rejoinder by the authors; Stat. Sci. 5, 403-434.

[13] A D Barbour and L H Y Chen: An Introduction to Stein's Method; Lecture Notes Series, Institute for Mathematical Sciences, National University of Singapore, Vol. 42005.

[14] L Barreira and B Saussol: Hausdorff dimension of measures via Poincaré recurrence; Comm. Math. Phys. 219 (2001) 443-463.

[15] R Bowen: Equilibrium States for Anosov Diffeomorphism; Springer Lecture Notes 470, Springer, New York/Berlin 1975.

[16] R C Bradley: Basic Properties of Strong Mixing Conditions. A Survey and Some Open Questions; Probability Surveys 2 (2005), 107-144.

[17] H Bruin, B Saussol, S Troubetzkoy and S Vaienti: Return time statistics via inducing; Ergod. Th. \& Dynam. Syst. 23, 991-1013 (2003).

[18] H Bruin and S Vaienti: Return times for unimodal maps; submitted to Forum Math.

[19] V Chamoître and M Kupsa: k-limit laws of return and hitting times; Discrete and Continuous Dynamical Systems 15 (2006), 73-86.

[20] J-R Chazottes, Z Coelho and P Collet: Poisson processes for subsystems of finite type in symbolic dynamics; Stochastics and Dynamics 9 (2009), 393-422.

[21] J-R Chazottes and P Collet: Poisson approximation for the number of visits to balls in nonuniformly hyperbolic dynamical systems; Ergod. Th. \& Dynam. Syst. 33 (2013), 49-80.

[22] J-R Chazottes and E Ugalde: Entropy estimation and fluctuations of hitting and recurrence times for Gibbsian sources; Disc. Cont. Dynam. Syst. Series B 5 (2005), 565-586.

[23] Z Coelho: Asymptotic laws for symbolic dynamical processes: In Topics in Symbolic Dynamics and Applications, edited by F Blanchard, A Maass and A Nogueira, LMS Lecture Notes Series 279, Cambridge University Press 2000, 123-165.

[24] Z Coelho and P Collet: Poisson law associated to subsystems of finite type in symbolic dynamical systems;

[25] P Collet: Some ergodic properties of maps of the interval; Dynamical Systems (Temuco, 1991/1992), Travaux en Cours, Vol. 52. Hermann Paris (1996) 55-91.

[26] P Collet: Statistics of closest return times for some non uniformly hyperbolic systems; Ergod. Th. \& Dynam. Sys. 21 (2001) 401-420.

[27] P Collet, A Galves and B Schmitt: Fluctuations of repetition times for Gibbsian sources; Nonlinearity 12 (1999), 1225-1237.

[28] K L Chung: A note on the ergodic theorem of information theory; Ann. Math. Stat. 32 (1961), $612-614$.

[29] M Denker: Remarks on weak limit laws for fractal sets; Progress in Probability Vol. 37, Birkhäuser 1995, 167-178.

[30] M Denker, M Gordin and A Sharova: A Poisson limit theorem for toral automorphisms; Illinois J. Math. 48(1) (2004), 1-20.

[31] W Doeblin: Remarques sur la théorie métrique des fraction continues; Compositio Mathematica 7 (1940), 353-371.

[32] V. M. Deschamps, B. Schmitt, M. Urbanski and A. Zdunik: Pressure and Recurrence; Fund. Math. 178 (2003), 129-141.

[33] P Doukhan: Mixing: Properties and examples; Lecture Notes in Statistics 85, Springer 1995.

[34] T Downarowicz: Entropy in Dynamical Systems; Cambridge University Press 2011.

[35] T Downarowicz and Y Lacroix: The law of series; Ergod. Th. \& Dynam. Syst. 31 (2011), 351-367.

[36] T Downarowicz, Y Lacroix and D Leandri: Spontaneous clustering in theoretical and some empirical stochastic processes, ESAIM Probab. Statist. 14 (2010), 256-262. 
[37] A Galves and B Schmitt: Inequalities for hitting times in mixing dynamical systems; Random Comput. Dynam. 5 (1997), 337-347.

[38] C Gupta, N Haydn, M Ko and A Rada: Entrance Time Convergence and Entropy; preprint USC 2012.

[39] A Freitas, J Freitas and M Todd: Hitting Time statistics and extreme value theory; Probab. Theory Related Fields 147 (2010), 675-710.

[40] A Freitas, J Freitas and M Todd: Extreme value laws in dynamical systems for non-smooth observations; J. Stat. Phys. 142 (2011), 108-126.

[41] A Freitas, J Freitas and M Todd: The compound Poisson limit ruling periodic extreme behaviour of non-uniformly hyperbolic dynamics; 2012 available at http://arxiv.org/abs/1204.2304.

[42] N Haydn: The Distribution of the first return time for rational maps; J. Stat. Phys. 94 (1999), $1027-1036$.

[43] N Haydn: Statistical properties of equilibrium states for rational maps; Ergod. Th. \& Dynam. Syst. 20 (2000), 1371-1390.

[44] N Haydn: A note on the limiting entry and return times distributions for induced maps; submitted to BIRS conference proceedings, available at http://arxiv.org/abs/1208.6059.

[45] N Haydn and Y Psiloyenis: Return times distribution for Markov towers with decay of correlations; preprint available at http://arxiv.org/abs/1010.1029.

[46] N Haydn, Y Lacroix and S Vaienti: Hitting and Return Times in Ergodic Dynamical Systems: Ann. of Probab. 33 (2005), 2043-2050.

[47] N Haydn, E Lunedei and S Vaienti: Averaged Number of Visits; Chaos 17 (2007), 033119.

[48] N Haydn and S Vaienti: The limiting distribution and error terms for return times of dynamical systems; Disc. Cont. Dyn. Syst. 10 (2004) 589-616.

[49] N Haydn and S Vaienti: The distribution of return times near periodic orbits; Probability Theory and Related Fields 144 (2009), 517-542.

[50] M Hirata: Poisson law for Axiom A diffeomorphisms; Ergod. Th. \& Dynam. Syst. 13 (1993), 533556.

[51] M Hirata: Poisson law for the dynamical systems with the "self-mixing" conditions; Dynamical Systems and Chaos, Vol. 1 (Worlds Sci. Publishing, River Edge, New York (1995), 87-96.

[52] M Hirata, B Saussol and S Vaienti: Statistics of return times: a general framework and new applications; Comm. Math. Phys. 206 (1999), 33-55.

[53] M Kac: On the notion of recurrence in discrete stochastic processes; Bull. A.M.S. 53 (1947),10021010.

[54] Y Kifer: Nonconventional Poisson limit theorems; preprint 2011 available at http://arxiv.org/abs/1110.2155v1.

[55] Y Kiefer and A Rapaport: Poisson and compound Poisson approximations in a nonconventional setup; preprint 2012, available at http://arxiv.org/abs/1211.5238.

[56] M Ko: Rényi Entropy and Recurrence; to appear in Disc. Cont. Dynam. Syst. 2012.

[57] I Kontoyiannis: Asymptotic Recurrence and Waiting Times for Stationary Processes; J. Theor. Prob. 11 (1998), 795-811.

[58] M Kupsa and Y Lacroix: Asymptotics for hitting times, Ann. of Probab. 33(3) (2005), 610-614.

[59] M Kupsa Example with non exponential return times.

[60] Y Lacroix: Possible limit laws for entrance times of an ergodic aperiodic dynamical system; Israel J. Math. 132 (2002), 253-264.

[61] R Mañé: Ergodic Theory and Differentiable Dynamics; Springer, 1987.

[62] A Nobel and A Wyner: A Recurrence Theorem for Dependent Processes with Applications to Data Compression; IEEE Vol. 38 No. 5 (1992), 1561-1564.

[63] Ornstein and Weiss; Entropy and Data Compression Schemes; IEEE Transactions on Information Theory 39 (1993), 78-83.

[64] Ornstein and Weiss; Entropy and Recurrence Rates for Stationary Random Fields; IEEE Transactions on Information Theory 48(6) (2002), 1694-97.

[65] K Petersen: Ergodic Theory: Cambridge studies in advanced mathematics \#2, 1983. 
[66] B Pitskel: Poisson law for Markov chains; Ergod. Th. \& Dynam. Syst. 11 (1991), 501-513.

[67] H Poincaré: Sur le problemèm des trois corps et les équations de la dynamique; Acta Mathematica 13 (1890), 1-270.

[68] Y Psiloyenis: Mixing Conditions and Return Times on Markov Towers; PhD thesis, USC, 2008.

[69] M Rosenblatt: A central limit theorem and a strong mixing condition; Proc. Nat. Acad. Sci. USA 42 (1956), 43-47.

[70] M Rosenblatt: Markov Processes. Structure and Asymptotic Behavior; Springer, Grundlagen \#184, 1971.

[71] J Rousseau and B Saussol: Poincaré recurrence for observations; Trans. AMS 362 (2010), 5845-5859

[72] B Saussol: Recurrence rate in rapidly mixing dynamical systems; Disc. Cont. Dynam. Sys. 15 (2006) 259-267.

[73] B A Sevast'yanov: Poisson limit law for a scheme of sums of independent random variables; Th. Prob. Appl. 17 (1972), 695-699.

[74] P Shields: Waiting Times: Positive and Negative Results on the Wyner-Ziv Problem; Theoretical Prob. 6 (1992), 499-519.

[75] H Wang, M Tang and R Wang: A Poisson limit theorem for a strongly ergodic non-homogeneous Markov chain; J. Math. Analysis Applications 277 (2003), 722-730.

[76] K Wasilewska: Limiting distribution and error terms for the number of visits to balls in mixing dynamical systems; PhD thesis USC 2013.

[77] L-S Young: Statistical properties of dynamical systems with some hyperbolicity; Annals of Math. 7 (1998), 585-650.

[78] L-S Young: Recurrence time and rate of mixing; Israel J. of Math. 110 (1999), 153-188. 